\title{
Effect of CME Interactions on the Production of Solar Energetic Particles
}

\author{
N. Gopalswamy*, S. Yashiro ${ }^{\dagger *}$, G. Michalek ${ }^{\dagger *}$, M. L. Kaiser*, R. A. Howard**, \\ R. Leske ${ }^{\ddagger}$ T. von Rosenvinge* and D. V. Reames* \\ ${ }^{*}$ NASA Goddard Space Flight Center, Greenbelt, MD 20771. \\ ${ }^{\dagger}$ Catholic University of America, Washington, DC 20064 \\ ${ }^{* *}$ Naval Research Laboratory, Washington, DC 20375. \\ ${ }^{\sharp}$ California Institute of Technology, Pasadena, CA 91125.
}

\begin{abstract}
.
We analyzed a set of 52 fast and wide, frontside western hemispheric (FWFW) CMEs in conjunction with solar energetic particle (SEP) and radio burst data and found that 42 of these CMEs were associated with SEPs. All but two of the 42 SEP-associated FWFW CMEs (95\%) were interacting with preceding CMEs or dense streamers. Most of the remaining 10 SEP-poor FWFW CMEs had either insignificant or no interaction with preceding CMEs or streamers, and were ejected into a tenuous corona. There is also a close association between type II radio bursts in the near-Sun interplanetary medium and SEP-associated FWFW CMEs suggesting that electron accelerators are also good proton accelerators.
\end{abstract}

\section{INTRODUCTION}

Copious production of nonthermal electrons and ions is an important aspect of solar eruptive events such as flares and coronal mass ejections (CMEs). Nonthermal electrons are inferred from the radio signatures they produce or detected by in situ observations. On the other hand, energetic ions need to be observed only in situ. Spectacular radio signatures indicating the production of nonthermal electrons at several solar radii from the Sun have been recently identified and found to be the result of colliding CMEs [1,2]. A natural question would be whether such CME interactions affect the production of nonthermal ions, commonly referred to as solar energetic particles (SEPs). A preliminary statistical study by Gopalswamy et al. [3, hereinafter Paper 1] suggests that a vast majority of the large SEP events are associated with fast CMEs that interacted with one or more preceding CMEs within about 20 solar radii. Within the current paradigm that CME-driven shocks [see, e.g., 4, 5] accelerate the SEPs from the upstream solar wind material, one would expect that the shocks pass through the material of the preceding CMEs. CMEs contain plasma structures with temperatures ranging from a few thousand $\mathrm{K}$ to several million $\mathrm{K}$, so the nature of the accelerated particles will depend on the physical properties of the source material that enters the shock. There are good indications that the composition of the SEPs significantly differs from that of the solar wind [see, e.g. 6]. Therefore, the sta- tistical association between CME interaction and large SEP events may be important in understanding the real situation in the acceleration sites of SEPs. One aspect of the statistical analysis in Paper 1 was to perform an inverse study of all the fast and wide, frontside western hemispheric (FWFW) CMEs to check their level of interaction with other CMEs and their association with SEPs. The inverse study confirmed the importance of CME interaction for SEP production. However, we did not examine the SEP-poor FWFW CMEs and the SEPassociated FWFW CMEs with no interactions in detail. In this paper, we report on our further analysis of the FWFW CMEs.

\section{DATA}

The FWFW CMEs observed by the Solar and Heliospheric Observatory (SOHO) mission's Large Angle and Spectrometric Coronagraph (LASCO) were selected from the SOHO/LASCO CME catalog maintained on line (http://cdaw. gsfc.nasa.gov/) by requiring that: 1 . the CME speed (V) is $>900 \mathrm{~km} / \mathrm{s}, 2$. the width $(\mathrm{W})>60^{\circ}, 3$. the CME span includes position angle $270^{\circ}$, and 4 . the solar source of the CME has a longitude between W0 and W90. During the period January 1996 to December 2001, we identified 52 CMEs, whose basic properties are listed in Table 1 (Date, UT, speed $(\mathrm{km} / \mathrm{s}), \mathrm{W}$, and solar source in columns $1-5)$. We 
TABLE 1. Characteristics of the FWFW events

\begin{tabular}{|c|c|c|c|c|c|c|c|c|}
\hline \multirow[b]{2}{*}{ Date } & \multicolumn{5}{|c|}{ CME } & \multirow[t]{2}{*}{ Type II } & \multicolumn{2}{|r|}{ SEP } \\
\hline & Time & Speed & Width & Location & Int & & I & Time \\
\hline $96 / 07 / 12$ & $15: 37$ & 1085 & 68 & S10W80 & $\mathrm{N}$ & $\mathrm{nN}$ & $\mathrm{N}$ & \\
\hline $96 / 11 / 28$ & $16: 50$ & 984 & 101 & N05W90 & F1 & $\mathrm{nN}$ & $\mathrm{e}$ & 21:00 \\
\hline $97 / 11 / 06$ & $12: 10$ & 1556 & $\mathrm{H}$ & S18W63 & F1 & $\mathrm{yY}$ & M & $12: 30$ \\
\hline $98 / 04 / 20$ & $10: 07$ & 1863 & 165 & S43W90 & F1 & $\mathrm{yY}$ & $\mathrm{M}$ & $11: 30$ \\
\hline $98 / 05 / 02$ & $14: 06$ & 938 & $\mathrm{H}$ & S15W 15 & NH & $\mathrm{yY}$ & M & $14: 00$ \\
\hline $98 / 05 / 06$ & $08: 29$ & 1099 & 190 & S11W65 & $\mathrm{F}^{*}{ }^{*}$ & $\mathrm{yY}$ & M & $08: 30$ \\
\hline $98 / 05 / 09$ & $03: 35$ & 2331 & 178 & N26W90 & $\mathrm{F} 1^{*}$ & $\mathrm{yY}$ & M & 05:00 \\
\hline $98 / 06 / 16$ & $18: 27$ & 1484 & 281 & S17W90 & $\mathrm{F}^{*} *$ & yY & $\mathrm{m}$ & $21: 30$ \\
\hline $98 / 11 / 05$ & $20: 44$ & 1118 & $\mathrm{H}$ & $\mathrm{N} 22 \mathrm{~W} 18$ & F1 & nY & $\mathrm{m}$ & $03: 00^{n}$ \\
\hline $99 / 06 / 04$ & $07: 26$ & 2230 & 150 & N17W69 & F1? & $\mathrm{yY}$ & M & $08: 30$ \\
\hline $99 / 06 / 24$ & $13: 31$ & 975 & $\mathrm{H}$ & N29W13 & F1 & $\mathrm{nN}$ & $\mathrm{e}$ & $04: 30^{n}$ \\
\hline $99 / 06 / 28$ & $21: 30$ & 1083 & $\mathrm{H}$ & $\mathrm{N} 22 \mathrm{~W} 44$ & $\mathrm{P}^{*}$ & yN & $\mathrm{N}$ & \\
\hline $99 / 07 / 25$ & $13: 31$ & 1389 & $\mathrm{H}$ & N38W81 & F1? & $\mathrm{yN}$ & $\mathrm{N}$ & \\
\hline $99 / 08 / 28$ & $01: 26$ & 1147 & 98 & S26W16 & P1 & $\mathrm{nN}$ & $\mathrm{N}$ & \\
\hline $99 / 09 / 21$ & 03:30 & 1402 & 125 & N19W90 & P1* & $\mathrm{yN}$ & $\mathrm{N}$ & \\
\hline $99 / 09 / 23$ & $15: 54$ & 1150 & 77 & S14W47 & P1? & $\mathrm{nN}$ & $\mathrm{N}$ & \\
\hline $00 / 02 / 09$ & $19: 54$ & 910 & $\mathrm{H}$ & S17W40 & $\mathrm{F} 1^{*}$ & $\mathrm{nN}$ & $\mathrm{N}$ & \\
\hline $00 / 02 / 12$ & $04: 31$ & 1107 & $\mathrm{H}$ & N26W 23 & $\mathrm{~F}^{*} *$ & yY & $\mathrm{m}$ & 06:00 \\
\hline $00 / 04 / 04$ & $16: 32$ & 1188 & $\mathrm{H}$ & N16W66 & $\mathrm{F} 2$ & $\mathrm{yY}$ & M & 17:00 \\
\hline $00 / 04 / 23$ & $12: 54$ & 1187 & $\mathrm{H}$ & N12W90 & $\mathrm{N}$ & $\mathrm{nN}$ & $\mathrm{m}$ & $15: 00$ \\
\hline $00 / 04 / 27$ & $14: 30$ & 1110 & 138 & $\mathrm{~N} 32 \mathrm{~W} 90$ & $\mathrm{~F}^{*}{ }^{*}$ & $\mathrm{nY}$ & $\mathrm{e}$ & $17: 00$ \\
\hline $00 / 05 / 04$ & $11: 26$ & 1404 & 170 & S17W90 & F2 & $\mathrm{yY}$ & $\mathrm{e}$ & $16: 30$ \\
\hline $00 / 05 / 15$ & $16: 26$ & 1212 & 165 & S24W67 & $\mathrm{F}^{*} *$ & $\mathrm{nY}$ & $\mathrm{m}$ & 19:00 \\
\hline $00 / 06 / 10$ & $\begin{array}{l}17: 08 \\
\text { 10: }\end{array}$ & 1108 & $\mathrm{H}$ & N22W 38 & $\mathrm{~F} 2 *$ & $\mathrm{yY}$ & M & $17: 30$ \\
\hline $00 / 06 / 15$ & $20: 06$ & 1081 & 116 & N20W65 & NS & $\mathrm{yY}$ & $\mathrm{e}$ & $01: 00^{n}$ \\
\hline $00 / 06 / 25$ & $07: 54$ & 1617 & 165 & N16W55 & P1 & $\mathrm{yY}$ & $\mathrm{m}$ & $11: 30$ \\
\hline $00 / 06 / 28$ & $19: 31$ & 1198 & 134 & N20W90 & $\mathrm{F} 1$ ? & $\mathrm{yN}$ & $\mathrm{e}$ & 20:00 \\
\hline $00 / 07 / 14$ & $10: 54$ & 1674 & $\mathrm{H}$ & N22W07 & $\mathrm{F}^{*} *$ & $\mathrm{yY}$ & M & $10: 30$ \\
\hline $00 / 07 / 22$ & $11: 54$ & 1230 & 105 & N14W56 & F1* & $\mathrm{yY}$ & M & $11: 30$ \\
\hline $00 / 08 / 11$ & $07: 31$ & 1071 & 70 & N27W90 & P1 & $\mathrm{nY}$ & M & $12: 00$ \\
\hline $00 / 09 / 12$ & $11: 54$ & 1550 & $\mathrm{H}$ & S17W09 & $\mathrm{F} 1 *$ & yY & $\mathrm{M}$ & 13:00 \\
\hline $00 / 10 / 16$ & $07: 27$ & 1336 & $\mathrm{H}$ & N05W90 & F2* & $\mathrm{yY}$ & M & 08:00 \\
\hline $00 / 11 / 08$ & 23:06 & 1345 & $\mathrm{H}$ & N10W77 & $\mathrm{F}^{*} *$ & $\mathrm{nY}$ & M & $23: 30$ \\
\hline $00 / 11 / 24$ & $05: 30$ & 994 & $\mathrm{H}$ & N20W05 & F2* & $\mathrm{yY}$ & $\mathrm{m}$ & $06: 00$ \\
\hline $00 / 11 / 24$ & $15: 30$ & 1245 & $\mathrm{H}$ & N22W07 & $\mathrm{F} 2 *$ & $\mathrm{yY}$ & M & $15: 30$ \\
\hline $01 / 01 / 28$ & $15: 54$ & 916 & 250 & S04W59 & NS & $\mathrm{nY}$ & M & $16: 30$ \\
\hline $01 / 02 / 10$ & 05:54 & 956 & $\mathrm{H}$ & N30W07 & $\mathrm{N}$ & $\mathrm{nN}$ & $\mathrm{N}$ & \\
\hline $01 / 02 / 11$ & $01: 31$ & 1183 & $\mathrm{H}$ & N24W57 & F2 & $\mathrm{yY}$ & $\mathrm{m}$ & $02: 30$ \\
\hline $01 / 03 / 29$ & $10: 26$ & 942 & $\mathrm{H}$ & N20W19 & $\mathrm{F} 1$ & $\mathrm{yY}$ & M & 12:00 \\
\hline $01 / 04 / 02$ & $11: 26$ & 992 & 80 & N17W60 & F1 & $\mathrm{yY}$ & $\mathrm{m}$ & $12: 00$ \\
\hline $01 / 04 / 02$ & $22: 06$ & 2505 & 244 & S19W72 & $\mathrm{F} 1^{*}$ & $\mathrm{yY}$ & M & 23:00 \\
\hline $01 / 04 / 09$ & $15: 54$ & 1192 & $\mathrm{H}$ & S21W04 & $\mathrm{N}$ & $\mathrm{yY}$ & $\mathrm{m}$ & $16: 00$ \\
\hline $01 / 04 / 10$ & $05: 30$ & 2411 & $\mathrm{H}$ & S23W09 & F1 & $\mathrm{yY}$ & M & $07: 30$ \\
\hline $01 / 04 / 12$ & $10: 31$ & 1184 & $\mathrm{H}$ & S19W43 & NS & $\mathrm{yY}$ & M & $11: 30$ \\
\hline $01 / 04 / 15$ & $14: 06$ & 1199 & 167 & S20W85 & F3 & $\mathrm{yY}$ & M & $14: 00$ \\
\hline $01 / 04 / 26$ & $12: 30$ & 1006 & $\mathrm{H}$ & N17W31 & F1? & $\mathrm{yY}$ & M & 14:00 \\
\hline $01 / 05 / 07$ & $12: 06$ & 1223 & 205 & N25W35 & $\mathrm{F} 2$ & $\mathrm{nY}$ & M & $13: 30$ \\
\hline $01 / 06 / 20$ & $19: 54$ & 1407 & $\mathrm{H}$ & N08W17 & $\mathrm{N}$ & $\mathrm{nN}$ & $\mathrm{N}$ & \\
\hline $01 / 07 / 19$ & $10: 30$ & 1668 & 166 & S08W62 & $\mathrm{N}$ & $\mathrm{nN}$ & $\mathrm{N}$ & \\
\hline $01 / 10 / 01$ & $05: 30$ & 1405 & $\mathrm{H}$ & S20W90 & $\mathrm{F} 4$ & nY & M & $13: 30$ \\
\hline $01 / 10 / 19$ & $16: 50$ & 901 & $\mathrm{H}$ & N15W29 & P1* & $\mathrm{yY}$ & M & $17: 30$ \\
\hline $01 / 11 / 04$ & $16: 35$ & 1810 & $\mathrm{H}$ & N06W18 & NS & $\mathrm{yY}$ & M & $16: 30$ \\
\hline
\end{tabular}

examined the GOES proton data for possible association of the FWFW CMEs with SEP events. If an SEP event was associated, we have given the intensity level (I) in the $>10 \mathrm{MeV}$ energy range and the onset times in columns 8 and 9, respectively (A superscript $n$ denotes the time corresponds to the next day). On the basis of the observed peak intensity (I), each event is classified as a major (M, with $\mathrm{I}>10 \mathrm{pfu})$, minor (m, with $1 \mathrm{pfu}<\mathrm{I}<10 \mathrm{pfu}$ ) or marginal (e, with $\mathrm{I}<1 \mathrm{pfu})$ event. The marginal events are those, which clearly stand above the noise level, but had an intensity $<1$ pfu. We also examined the association of the CMEs with metric type II bursts from the online Solar Geophysical Data (SGD) and interplanetary type II bursts from the Wind/WAVES catalog (http: / / lep694.gsfc. nasa.gov/waves/waves.html). These data will tell us whether the CMEs were responsible for electron acceleration. The presence $(y, Y)$ or absence $(n, N)$ of type II radio bursts are indicated in column 7, with small (capital) letters referring to metric (DH) type II bursts. Using movies of SOHO/LASCO images and the CME catalog, we identified potential instances of interaction between
TABLE 2. FWFW CMEs, Interactions, and SEP Association

\begin{tabular}{lrr}
\hline & With SEPs & without SEPs \\
\hline No interaction & $7(2)$ & 4 \\
Interaction & $35(40)$ & 6 \\
\hline
\end{tabular}

the FWFW CMEs and other preceding CMEs. This information is given in column 6. If the extent of position angle (PA) overlap between FWFW CMEs and the preceding ones is $>30^{\circ}$, the interaction is characterized as full $(\mathrm{F})$, and partial $(\mathrm{P})$ otherwise. The number of preceding CMEs is also indicated as a subscript to F and P. An * in column 7 means there were additional interactions, and a ? means the interaction was beyond 30 solar radii, but within 50 solar radii. NL and NS denote that there was no intersection of trajectories, but there was interaction with the leg of a preceding CME (NL) or with a dense streamer (NS). One event (1998 may 02, marked $\mathrm{NH}$ in column 6) with no apparent interaction was preceded by halo CMEs and hence might have interacted with them along the line of sight.

\section{ANALYSIS AND RESULTS}

The first thing we notice in Table 1 is that 42/52 (81\%) of the FWFW CMEs were associated with SEP events identified from GOES data. For the remaining 10 events (19\%), we did not find an SEP event above the noise level in the GOES proton plots. Table 2 gives the extent of CME interaction and the SEP association for the 42 events. For simplicity, we have combined the full and partial interactions together as simply "interactions". Similarly, we did not distinguish an SEP event whether it is a major, minor, or marginal event. This is roughly the level of association found in the study of SEPs in Paper 1. It is clear that the lower left cell in Table 2 is the dominant one, suggesting that $83 \%$ of all FWFW CMEs that had SEPs were preceded by CME interaction, similar to the level of interaction found starting from SEP events in Paper 1.

TABLE 3. SEP-associated CMEs without interaction

\begin{tabular}{lrrrl}
\hline Date & $\mathrm{V}(\mathrm{km} / \mathrm{s})$ & $\mathrm{W}(\mathrm{deg})$ & Location & Type \\
\hline $98 / 05 / 02$ & 938 & 360 & $\mathrm{~S} 15 \mathrm{~W} 15$ & Major, NH \\
$00 / 04 / 23$ & 1187 & 360 & N12W90 & minor \\
$00 / 06 / 15$ & 1081 & 116 & N20W65 & marginal, NS \\
$01 / 01 / 28$ & 916 & 250 & S04W59 & Major, NS \\
$01 / 04 / 09$ & 1192 & 360 & S21W04 & minor \\
$01 / 04 / 12$ & 1184 & 360 & S19W43 & Major, NS \\
$01 / 11 / 04$ & 1810 & 360 & N06W18 & Major, NS \\
\hline
\end{tabular}


TABLE 4. SEP-poor CMEs with Interaction

\begin{tabular}{lrrrl}
\hline Date & $\mathrm{V}(\mathrm{km} / \mathrm{s})$ & $\mathrm{W}(\mathrm{deg})$ & Location & Type \\
\hline $99 / 06 / 28$ & 1083 & $360(28)$ & N22W44 & N/P TB \\
$99 / 07 / 25$ & 1389 & $360(97)$ & N38W81 & N/F TB \\
$99 / 08 / 28$ & 1147 & $98(12)$ & S26W16 & N/P TB \\
$99 / 09 / 21$ & 1402 & $125(14)$ & N19W90 & N/P TB \\
$99 / 09 / 23$ & 1150 & $77(9)$ & S14W47 & N/P TB \\
$00 / 02 / 09$ & 910 & $360(37)$ & S17W40 & N/F TB \\
\hline
\end{tabular}

\section{SEP-associated CMEs without Interaction}

We now look at the $17 \%$ of the FWFW CMEs that were associated with SEPs, but were apparently not preceded by CME interaction (see Table 3 ). Four of the seven FWFW CMEs without interaction had major SEPs. This is somewhat embarrassing because we have claimed that $\mathrm{CME}$ interaction is an important aspect of SEP production. However, LASCO movies showed that the primary CMEs in these events interacted with dense streamers, completely destroying them. Most streamers represent the pre-eruption manifestations of CMEs and have the same three-part structures as the CMEs. Thus, the interaction with the streamers should be similar to the interaction with other CMEs. Out of the seven FWFW CMEs in Table 3, four interacted with streamers (denoted by NS in the last column of Table 3). In the case of 1998 May 2 event, the FWFW CME in question was preceded by two halo CMEs from the same active region (noted as "NH" in Table 3). The height-time plots that describe the interaction correspond to the sky plane measurements, which generally underestimate CME speeds of halo CMEs. Therefore one cannot rule out interaction with preceding CMEs. Thus only two of the seven FWFW CMEs can be regarded to have no interactions with any confidence. Thus, allowing for interaction with dense streamers, we see that 40/42 (95\%) FWFW CMEs with SEPs interacted with one or more preceding CMEs, again consistent with Paper 1 . In the remaining 2 cases (5\%) without interaction, the SEP events were minor.

\section{SEP-Poor CMEs with Interaction}

If CME interaction is important for SEP production, why were the six FWFW CMEs in Table 2 (also listed in Table 4) that were preceded by CME interaction were not associated with any SEPs? In the last column of Table 4, N/P (N/F) means no SEP but there was partial (full) interaction. To answer this question, we examined the properties of the preceding CMEs. The most striking aspect of the preceding CMEs is that all but one were very narrow (see the number within parentheses in the W column of Table 4), there by reducing the extent of overlap to the width of the preceding CMEs (see Fig 1).

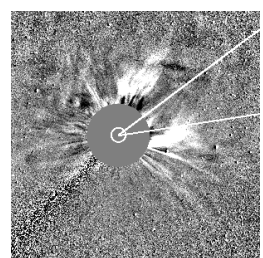

(a) $1999 / 06 / 28$ 23:18

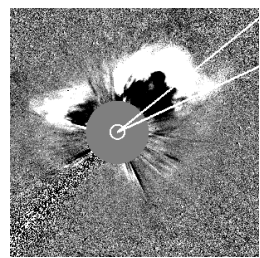

(d) $1999 / 09 / 2105: 18$

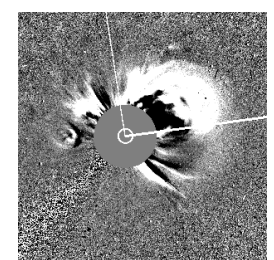

(b) $1999 / 07 / 2515: 18$

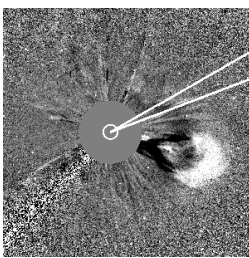

(e) $1999 / 09 / 2318: 18$

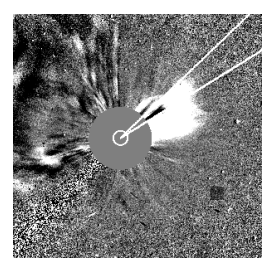

(c) $1999 / 08 / 28$ 02:42

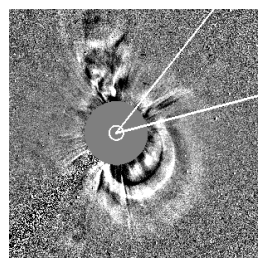

(f) $2000 / 02 / 0922: 18$
FIGURE 1. One SOHO/LASCO/C3 running difference image for each of the SEP-poor CMEs in Table 4. The width of the preceding CME is indicated by the two solid lines.

In Paper 1, the average extent of overlap between preceding and primary CMEs was found to be $\sim 50^{\circ}$. Thus, the interaction is not expected to be severe in all but one case. The small overlap between the primary and preceding CMEs also reduces the chance of the interaction region having a magnetic connection to the Earth. In the one case (1999 July 25), the extent of overlap is quite extensive, but there was another problem: even though the trajectories showed intersection, the preceding CME had departed the Sun some 17 hours earlier and faded to the background level even before the arrival of the primary CME. Thus in all the cases the severity of interactions is rather low and for practical purposes, the events in Table 4 must be considered as FWFW CMEs with no interaction.

\section{SEP-poor CMEs with No Interaction}

Finally, we examine the four FWFW CMEs without SEPs and without interaction (see Table 5). In the last column of Table 5, NN denotes no interaction at all and NE means interaction above eastern hemisphere, which is not relevant to SEPs. This is the crucial sample that supports our suggestion that CME interaction is an important aspect of SEP production. Unfortunately, the sample size is rather small. We need to collect more events of this kind to arrive at firmer conclusions. Nevertheless, it is instructive to examine the circumstances of these eruptions to gain some insight as to why these FWFW CMEs were SEP-poor. Figure 2 shows a LASCO/C3 snapshot of each of the four CMEs. The width of the first event was only slightly above average thus barely satisfying the width requirement to be classified as FWFW events. Two events are halos but there 
TABLE 5. SEP-poor CMEs with No interaction

\begin{tabular}{lrrrl}
\hline Date & V $(\mathrm{km} / \mathrm{s})$ & W $(\mathrm{deg})$ & Location & Type \\
\hline $96 / 07 / 12$ & 1085 & 68 & S10W80 & NN \\
$01 / 02 / 10$ & 956 & 360 & N30W07 & NE TB \\
$01 / 06 / 20$ & 1407 & 360 & N08W17 & NE TB \\
$01 / 07 / 19$ & 1668 & 166 & S08W62 & NN TB \\
\hline
\end{tabular}

is no way of knowing their true widths. The first event was close to the solar minimum and hence was confined to the extent of the equatorial streamer belt. In the other three events, the CMEs were ejected into a tenuous background (noted as TB in the last column of Table 5) compared to the opposite hemisphere. This is also the case with all the events in Table 4. The lower density means higher Alfven speed in the medium and weaker shocks. The situation is opposite to that of running into a dense structure such as a CME or a streamer.

\section{Electron and Proton Accelerations}

Metric and IP type II bursts are indicative of nonthermal electrons accelerated by MHD shocks. The same shocks may also accelerate protons and heavier ions. From Table 1, we found that 38/52 (73\%) of FWFW CMEs had DH type II bursts while 35/52 (67\%) had metric type II bursts. For the 42 SEP-associated FWFW CMEs, the association was better: $90 \%$ and $79 \%$ for $\mathrm{DH}$ and metric type II bursts, respectively. As was pointed out in Paper 1, SEP-poor FWFW CMEs were also electron-poor (no DH type II). However, three of the 10 SEP-poor FWFW CMEs were associated with the metric type II bursts. Three marginal and one minor SEP events were not associated with DH type II bursts (the 2000 June 25 event had a weak DH type II, but was listed as a no DH event in Paper 1), although the minor and one marginal SEP events had metric type II burst association. The difference between metric and DH type II bursts has been attributed to different radial profiles of the Alfven speed in the corresponding regions of the solar corona [7].

\section{DISCUSSION AND CONCLUSIONS}

The analysis presented in this paper confirms the importance of CME interaction in the production of SEPs. A detailed analysis of the SEP-poor FWFW CMEs with interaction reveals that the interactions are insignificant. Similarly, FWFW CMEs with SEPs but no apparent interaction with preceding CMEs had significant interaction with large-scale streamers. The extreme cases of FWFW CMEs with no interaction and no SEPs were
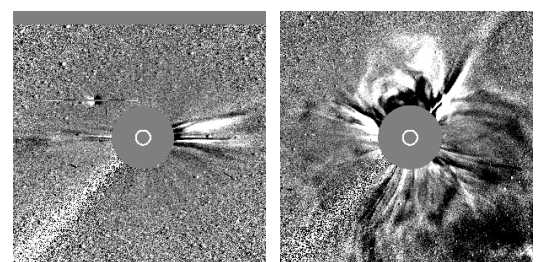

(a) $1996 / 07 / 1220: 56$
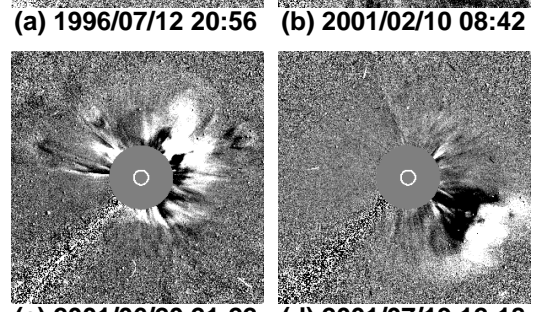

(c) $2001 / 06 / 2021: 22$

(d) $2001 / 07 / 1912: 18$

FIGURE 2. One SOHO/LASCO/C3 running difference image for each of the SEP-poor CMEs in Table 5.

ejected into very tenuous regions of the corona with no apparent structures to interact with. The close association between DH type II bursts and fast and wide CMEs reported elsewhere [8] is consistent with the results of this paper that electron accelerators are also proton accelerators. As we had pointed out before, the efficiency of particle acceleration is boosted when a fast shock runs into preceding CMEs. This might result in shock strengthening due to the enhanced density in the preceding CMEs as compared to the solar wind (provided the magnetic field is not enhanced significantly). Particles may also be trapped in the closed field lines of the preceding CMEs or the associated turbulence so they are subject to repeated acceleration by the shock for the time the shock takes to transit through the preceding CME. The preceding CMEs may also drive weak shocks that may preaccelerate suprathermal ions even if they are too weak to accelerate $>1 \mathrm{MeV}$ ions. What happens after the shock passage is an interesting problem: the main body of the fast CME comes in contact with the slower CME and might form a merged resultant $\mathrm{CME}$. We refer to this merger as CME cannibalism and needs further investigation.

\section{REFERENCES}

1. Gopalswamy, N. et al., ApJ, 548, L91, 2001a

2. Gopalswamy, N. et al., GRL, 29(8), 10.1029/2001GL013606, 2002a

3. Gopalswamy, N. et al., ApJ, 572, L103, 2002b

4. Reames, D. V., Space Sci. Rev., 90, 413, 1999

5. Mason, G. et al., ApJ, 525, L133, 1999.

6. Mewaldt, R. et al. 2002, Adv. Space Res., in press, 2002

7. Gopalswamy, N. et al., JGR, 106, 25261, 2001b

8. Gopalswamy, N. et al., JGR, 106, 29219, 2001c 